\title{
NONLINEARITY AND HYSTERESIS OF RESONANT STRAIN GAUGES
}

\author{
Chengqun Gui*, Rob Legtenberg, Harrie A. C. Tilmans**, Jan H. J. Fluitman, Miko Elwenspoek \\ MESA Research Institute, University of Twente, P.O.Box 217 \\ 7500 AE Enschede, the Netherlands
}

\begin{abstract}
Nonlinearity and hysteresis effects of electrostatically activated, voltage driven resonant microbridges have been studied theoretically and experimentally. It is shown, that, in order to avoid vibration instability and hysteresis to occur, the choices of the a.c. and d.c. driving voltages and of the quality factor of a resonator, with a given geometry and choice of materials, are limited by a hysteresis criterion. The limiting conditions are also formulated as hysteresis-free design rules. An expression for the maximum attainable figure of merit is also given. Experimental results, as obtained from electrostatically driven vacuum-encapsulated polysilicon microbridges, are presented and show good agreement with the theory.
\end{abstract}

\section{Introduction}

Non-linearities are easily encountered in resonant microbridges, leading to additional stiffning, known as the "hard spring effect", and, moreover, to hysteresis and instability to occur [1-6]. In particular the latter two phenomena, seriously limit the freedom of geometric design, the operating conditions, the maximum allowable quality factor, and subsequently the figure of merit. Nonlinearity of micromachined resonant microbridges has been observed and studied by several authors [2-9]. In 1987, M. V. Andres et al experimentally observed the nonlinear vibration and hysteresis of micromachined silicon resonators [2]. $\mathrm{K}$.

\footnotetext{
* On leave from: The Precision Instruments Department, Tsinghua University, Beijing, 100084, P. R. China.

"* Present address: Katholieke Universiteit, Departement Elektrotechniek-EAST, Kardinaal Mercierlaan 94, B-3001 Heverlee, Belgium
}

Ikeda et al discussed the influence of the nonlinear vibration of the $\mathrm{Si}$ resonant beam on the sensor characteristics, and introduced a method to eliminate this influence in 1989 [3]. J. D. Zook et al also found in 1991 that the micro-beam resonators are sensitive to the operating conditions: as the drive voltages increase, the resonant frequency shifts to a higher value, leading to hysteresis depending on the direction of frequency scan [4]. R. I. Pratt et al reported in 1991 that the nonlinear response curves of the lateral vibrating micromechanical structure are well modelled by Duffing's equation for a stiffening spring [5]. H.A.C. Tilmans et al. discussed the nonlinear large amplitudes effects and derived expressions for the stiffening effect and for the critical amplitude of resonant microbridges $[6,7,9]$. The formulations are based on the theory of a discrete stiffening spring as introduced by L.D. Landau and Lifshitz [10]

In this paper, we demonstrate a hysteresis criterion, a necessary condition for the hysteresis-free operation of the resonant microbridges. This hysteresis criterion depends on the quality factor, the operating, the geometric properties and material properties of the microresonator. The influence of hysteresis on the performance of the microbridges, such as the figure of merit, sensitivity and noise, are discussed. Hysteresisfree design rules associated with quality factors, the figure of merit, geometry and operating of the microresonator are addressed. Experimental results that show the validity of the hysteresis criterion are given.

\section{Hysteresis Criterion}

The resonant microbridge considered here consists of a prismatic wide beam with a rectangular cross section, having clamped end conditions. Axial stress effects, due to either a built-in strain field or to externally applied 
loads, are not taken into account. This is justified to some extent, recalling that axial stress effects are generally tensile in nature, which leads to a stiffer structure. This means that for such structures, the criteria as derived in this section are worst case conditions and as such define a safe operating region. The harmonic forced nonlinear vibration equation of the clamped-clamped beam has the same form as Duffing's equation (see equation (21) of [11]), which means that the microbridge can be approximated as a simple springmass system with a restoring force having a cubic dependence on the amplitude $[12,13]$. Thus the fundamental resonant frequency $\omega$ which depends on deflection can be written as [6]:

$\omega^{2}=\omega_{0}^{2}\left(1+0.53\left(1-v^{2}\right)\left(W_{\max } / h\right)^{2}\right)$

with $\omega_{0}$ the resonant frequency in the linear limit, $v$ Poisson's ratio and $W_{\max } / h$ the ratio of the maximum vibration amplitude to the thickness of the microbridge.

A consequence of equation (1) is that beyond a critical amplitude $W_{c}$ the resonant curve becomes triple valued, see figure 1 . The critical amplitude for the first mode is given by $[6,10]$ :

$W_{c}=\frac{h}{\left(0.53 Q_{1}\left(1-v^{2}\right)\right)^{1 / 2}}$

with $Q_{1}$ the quality factor for the first mode.

The maximum vibration amplitude $W_{\max }$ for the first mode of a resonator electrostatically driven by a $\mathbf{d c}$ polarization voltage $V_{p}$ plus an ac oscillating voltage $V_{\text {osc }}$ is [9]:

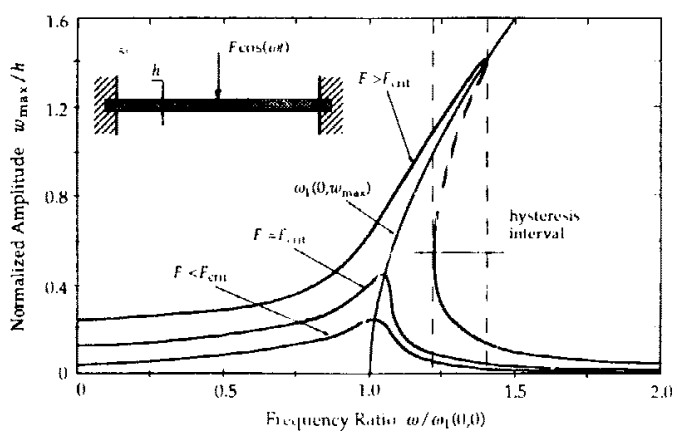

Figure 1, Normalized amplitude versus the normalized frequency for a clamped-clamped beam with a rectangular cross section and with zero built-in axial strain.
$W_{\max }=0.37 d Q_{1} \frac{V_{p} V_{o s c}}{V_{p i o}^{2}}$

with $d$ the gap spacing and $V_{p i o}$ is the pull-in voltage of a microbridge with a uniform electrod. An approximated expression of $V_{p o}$ is given by:

$V_{p i o}=3.48 \frac{1}{l 2}\left[E^{4} h^{3} d^{3} / \varepsilon_{0}\right]^{1 / 2}$

where $E^{t}=E /\left(1-v^{2}\right)$ is the plate modulus, $E$ is Young's modulus, $l$ denotes the beam length, $\varepsilon_{0}$ is the dielectric constant of vacuum $[7,9]$.

Obviously, for hysteresis-free operation, we must have $W_{\max }<W_{c}$, which leads to the hystersis criterion

$V_{p} V_{o s c} Q_{1}^{3 / 2}<K_{h c}$

where

$K_{h c}=47.0\left(\frac{E^{\prime}}{\varepsilon_{0}}\right)\left(\frac{h}{l}\right)^{4} d^{2}$

is a hysteresis critical constant that depends on the geometric properties and the material parameters of the microbridges.

Equation (5) represents a necessary condition for the hysteresis-free operation of a resonant microbridge. The hysteresis criterion for the driving voltages and the quality factor of a typical microbridge is graphically shown in figure 2 .

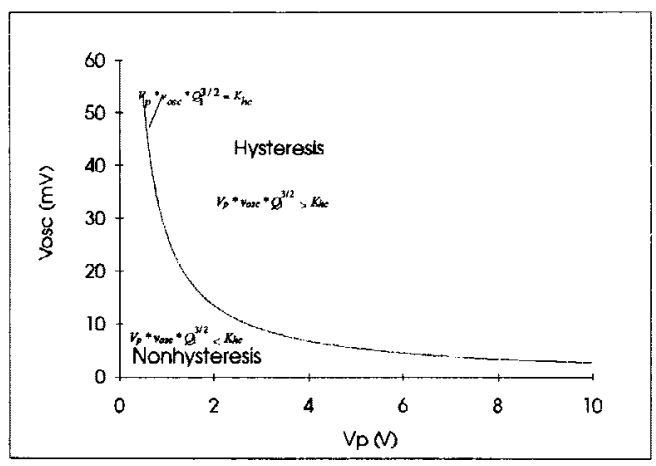

Figure 2, Hysteresis criterion of a typical encapsulated resonant microbridge. Resonator size is $210 X 100 X 1.5$ um, gap spacing is $1.0 \mu \mathrm{m}$, pull-in voltage is $28.0 \mathrm{~V}$, $Q_{1}=2700$ and plate modulus $E^{\prime \prime}=166 \mathrm{MPa}$ 
In general, the chance for vibration instability can be lowered, by choosing a smaller beam aspect ratio $l / h$ or by increasing the gap spacing. Also, recall, as indicated at the beginning of this section, that an axial tensile stress will make the beam stiffer, which leads to an increase of the pull-in voltage [9], and thus a higher value for $K_{h c}$.

\section{Hysteresis-free Design Rules}

The hysteresis criterion expressed by eqs. (5) and (6) leads to a complex interplay between the desire for a stable, hysteresis-free design and the best attainable performance of the microbridge expressed in terms of the figure of merit and of the (axial) strain sensitivity and resolution. In this section, eq. (5) will be used to show that the quality factor may not exceed a certain maximum value in order to avoid instability and hysteresis to occur. Also, the expression for a maximum attainable figure of merit will be derived. From eq. (6), it follows, that the maximum allowable quality factor $Q_{\text {max }}$ is given by:

$Q_{1 \max }=13.02\left(\frac{E}{\varepsilon_{0}}\right)^{2 / 3}\left[\frac{d^{2}}{V_{p} V_{o s c}}\left(\frac{l}{h}\right)^{4}\right]^{2 / 3}$

In the design of the micro resonant strain gauges, a high quality factor is prefered to attain a low energy dissipation, high frequency stability, high resolution and high efficient oscillator. In order to increase the freedom of the hysteresis-free quality factor, it is suggested in equation (7) to decrease the drive voltages and the aspect ratio, or increase the gap spacing. However, decreasing the drive voltages is limited by noise. The penalty of decreasing the aspect ratio of the beam has to be paid by a decreased sensitivity. The sensitivity of the resonant strain gauges is characterized by it's gauge factor (in case of a zero axial force) [6]:

$G_{\varepsilon 1}=0.5 \gamma_{1}\left(1-v^{2}\right)(l / h)^{2}$

with $G_{\varepsilon 1}$ the gauge factor, $\gamma_{1}=0.295$ the coefficient for the first mode. Obviously, as decreasing the aspect ratio of the resonant beam, the gauge factor will be decreased on the order of $(l / h)^{2}$.

The figure of merit is another relevant parameter that is much influenced by the hysteresis criterion. In an electromechanical microresonator, the electrostatically driven microbridge is coupled to a control circuit to obtain self-oscillation in the required mode. To achieve this, it is important to have a large figure of merit. The figure of merit, defined as the ratio of the motional and the static admittance, provides a measure that combines the quality factor and the coupling factor of the microresonator into one parameter [7]

$M \equiv \frac{\left|Y_{m o t}\left(\omega_{1}^{s}\right)\right|}{\left|Y_{\text {stat }}\left(\omega_{1}^{s}\right)\right|} \approx Q_{1} K_{e f f}^{2}$

with $M$ the figure of merit, $\left|Y_{\text {mot }}\left(\omega_{1}^{s}\right)\right| /\left|Y_{\text {stat }}\left(\omega_{1}^{s}\right)\right|$ is the ratio of the motional and the static admittance respectively, $K_{e f f}$ is the coupling factor of the microresonator. Based on the theory presented in $[7,9]$, the figure of merit can be conveniently expressed as a function of the drive voltages

$M \approx 0.20 Q_{1}\left(\frac{V_{p}}{V_{p i o}}\right)^{2}$

Introducing equation (5) into (10), we have

$M<M_{\max } \approx 3.01(h / d)^{2}\left(\frac{V_{p i o}}{V_{o s c} Q_{1}}\right)^{2}$

Interestingly, on the one hand, it is seen from equation (10) that the value of the figure of merit increases with increasing quality factor and with decreasing pull-in voltage. On the other hand, from a stability point of view (i.e., equation (11)), the allowed upper bound of the figure of merit will be smaller for higher values of the quality factor and for smaller values of the pull-in voltage. Also note, that low driving levels are beneficial for obtaining a high value for $M_{\max }$.

\section{Experimental and Results}

In order to verify the foregoing theory with experimental data, vacuum encapsulated polysilicon microbridge resonators have been designed and fabricated using the process flow as described in $[7,8]$. A SEM photograph of a completed device is shown in Fig. 3. Bridges of two different lengths $(210 \mu \mathrm{m}$ and $310 \mu \mathrm{m}$ ), with a width of $100 \mu \mathrm{m}$, a thickness of $1.5 \mu \mathrm{m}$ and a gap spacing of $1.0 \mu \mathrm{m}$ have been tested. The quality factor was set by controlling the cavity pressure during the reactive sealing step with LPCVD silicon nitride [14]. This way, the quality factor could be varied between 100 and 3000 .

The distorsion of the resonance curve and the hysteresis beyond $W_{c}$ due to nonlinearity can be obtained by measuring the admittance as a function of the frequency using a HP4194 gain-phase analyzer. While increasing the oscillating voltage, the resonant frequency shifts to a higher value, see figure 4,5 and 6 . The resonator will experience three conditions: a 
nonhysteresis region (figure 4), a critical point (figure 5) and a hysteresis region (figure $6 \mathrm{a}$ and $6 \mathrm{~b}$ )

The experimental results of the hysteresis criterion for resonators with a length of $210 \mu \mathrm{m}$ and $310 \mu \mathrm{m}$ are illustrated in figure 7 and figure 8, respectively. Both plots have shown the validity of equation (5). The mean error of experimental results less than $5 \%$.

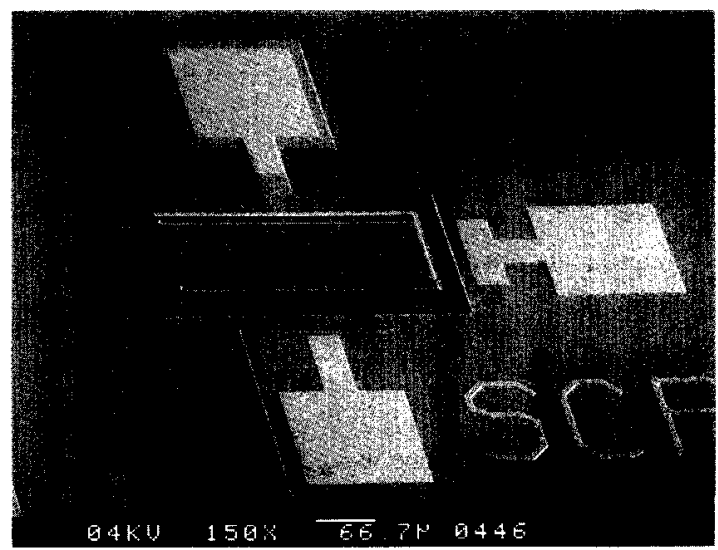

Figure 3, SEM photograph of cap and bondpads of the microbridge

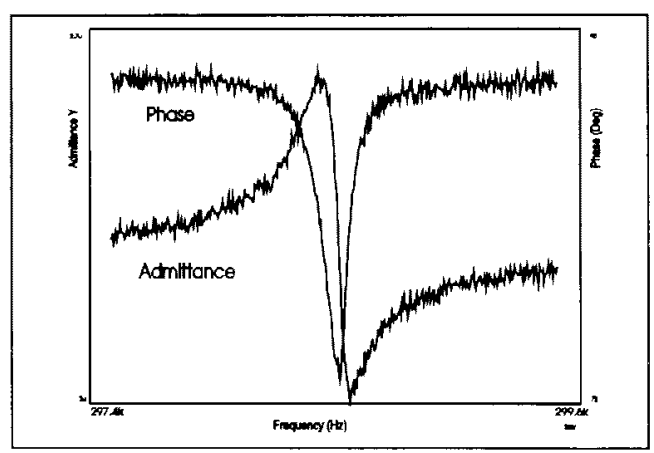

Figure 4, Admittance, phase vs frequency plot (nonhysteresis). Resonator No. 3209, size $210 X 100 X 1.5 \mu \mathrm{m}$, gap spacing $1.0 \mu \mathrm{m}, V_{p}=1 \mathrm{~V}$, $V_{\text {osc }}=10 \mathrm{mV}, Q_{1}=2871$

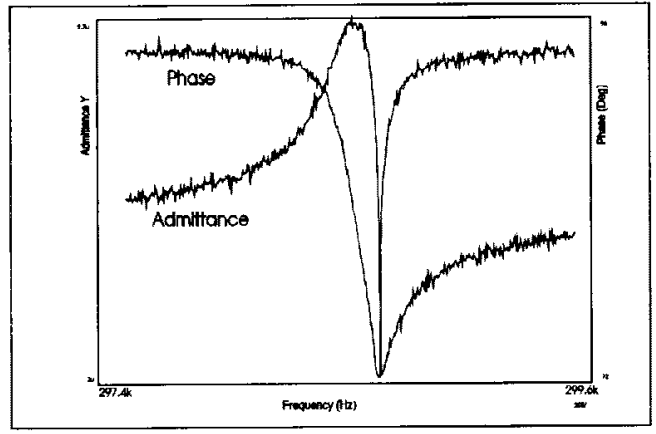

Figure 5, Admittance, phase vs frequence plot (at critical load). Resonator No. 3209, size $210 X 100 X 1.5 \mu \mathrm{m}$, gap spacing $1.0 \mu \mathrm{m}, V_{p}=1 \mathrm{~V}$, $V_{o s c}=15 \mathrm{mV}, Q_{1}=2871$

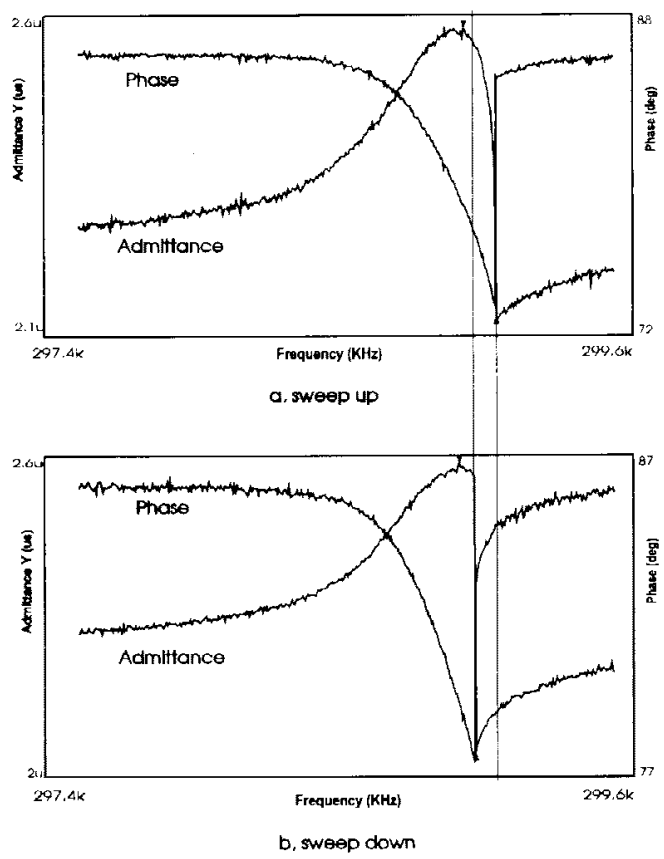

Figure 6, Admittance, phase vs frequence plot (hysteresis). Resonator No. 3209, size $210 X 100 X 1.5 \mu \mathrm{m}$, gap spacing $1.0 \mu \mathrm{m}, V_{p}=1 \mathrm{~V}$, $V_{\text {osc }}=20 \mathrm{mV}, Q_{1}=2871$ 


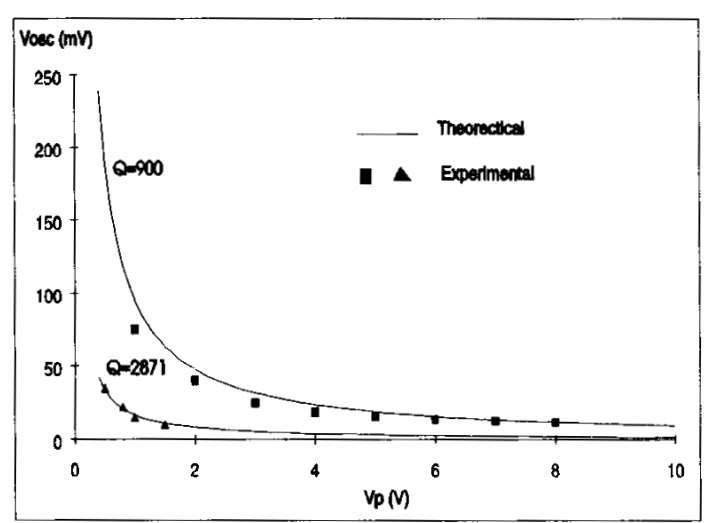

Figure 7, Hysteresis criterion. Resonator size $210 X 100 X 1.5 \mu \mathrm{m}$, Resonator No. 3209, $Q_{1}=2871$, Resonator No. 3220, $Q_{1}=900$. Higher quality factor means less freedom of microresonator.

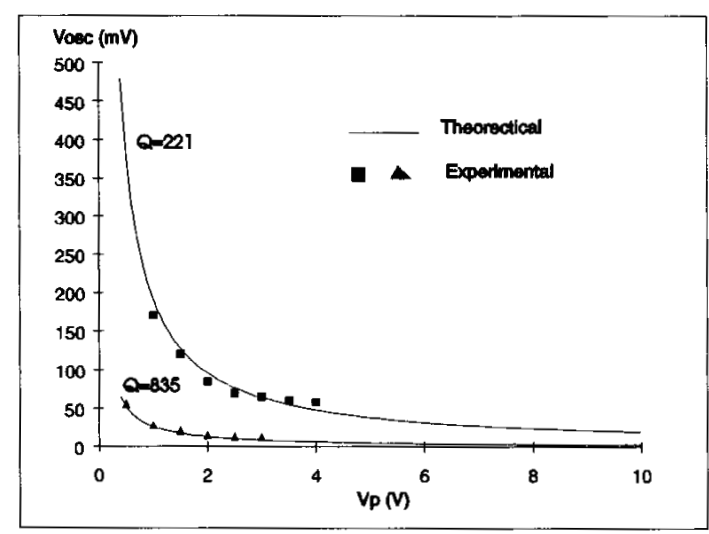

Figure 8, Hysteresis criterion. Resonator size $310 X 100 X 1.5 \mu \mathrm{m}$, Resonator No. 3304, $Q_{1}=835$, Resonator No. 5307, $Q_{1}=221$. Lower quality factor implies larger freedom of microresonator.

\section{Discussion and Conclusions}

Hysteresis and instability due to the hard spring effect of resonant microbridges have been studied. A hysteresis criterion, which depends on the quality factor, the operating and the geometry of the microresonator, has been obtained. This hysteresis criterion represents a necessary condition for the hysteresis-free operation of the resonant microbridge. It was found that the freedom of the hysteresis-free microresonator may be increased by decreasing the quality factor, the drive voltages and the aspect ratio of the beam, or increasing the pull-in voltage of the microbrdge. All these measures have their shortcoming: decreasing the quality factor will be accompanied with a loss of resolution and frequency stability, decreasing the drive voltages is limited by noise, decreasing the aspect ratio of the beam will cause the undesirable loss of sensitivity which is decreased on the order of $(l / h)^{2}$.

The figure of merit is limited by the hysteresis criterion. On the one hand, the value of the figure of merit will be increased by increasing the quality factor, on the other hand the nonhysteresis freedom of the figure of merit will be decreased by increasing the quality factor. For a given $Q$ factor, the figure of merit can be increased by increasing $V_{o s c}$ up to a maximum value bounded by hysteresis. Hysteresis-free design rules associated with quality factor, the figure of merit, operating and geometry of the microresonator, sufficiently demonstrate the complex interplay between stability and the performance of the resonant microbridges.

Hysteresis and instability are successfully detected by using a gain-phase analyzer. Experimental results of resonant microbridges with a length of $210 \mu \mathrm{m}$ and $310 \mu \mathrm{m}$ have shown the validity of the hysteresis criterion.

\section{Acknowledgements}

The authors acknowledge Meint de Boer and Erwin Berenschot for their help with the fabrication of the encapsulated resonant microbridges. Bert Otter is acknowledged for making the SEM photograph. This research is part of the programme of the Dutch Foundation for Fundamental Research on Matter (FOM) and is sponsored by the Dutch Technology Foundation (STW).

\section{References}

[1] M. Elwenspoek, Micromechanical resonant sensors, Journal A(1991) pp 15-21.

[2] M. V. Andres, K. W. H. Foulds and M. J. Tudor, Nolinear vibration and hysteresis of micromachined silicon resonators designed as frequency-out sensors, Electron. Lett. 23(1987) pp 952-954.

[3] K. Ikeda, H. Kuwayama, T. Kobayashi, T. Watanabe, T. Nishikawa, T. Yoshida and K. Harada, Study of nonlinear vibration silicon resonant beam strain gauges, Proc. of 8th Sensor Symp., Tokyo, Japan, 1989, pp.21-24.

[4] J. D. Zook, D. W. Burns, H. Guckel, J. J. Sniegowski, R. L. Engelstad and Z. Feng, Resonant microbeam strain transducers, Proc. 6th Int. Conf. 
Solid-State Sensors and Actuators (Transducers '91), San Francisco, U. S. A., June 24-27, 1991, pp. 529-532.

[5] R. I. Pratt, G. C. Johnson, R. T. Howe and J. C. Chang, Micromechanical structures for thin film characterization, Proc. 6th Int. Conf. Solid-State Sensors and Actuators (Transducers '9l), San Francisco, U. S. A., June 24-27, 1991, pp. 205-208.

[6] H. A. C. Tilmans, M. Elwenspoek and J. H. J. Fluitman, Micro resonant force gauges, Sensors and Actuators A, (1992) pp.35-53.

[7] H. A. C. Tilmans, Micro-mechanical Sensors Using Encapsulated Built-in Resonant Strain Gauges, PhD Thesis, University of Twente, 1993.

[8] R. Legtenberg, H. A. C. Tilmans, Electrostatically driven vacuum-encapsulated polysilicon resonators, part I, design and fabrication, Sensors and Actuators A 45, (1994) pp.57-66.

[9] H. A. C. Tilmans, R. Legtenberg, Electrostatically driven vacuum-encapsulated polysilicon resonators, part II, theory and performance, Sensors and Actuators A 45, (1994) pp.67-84.

[10] L. D. Landau and E. M. Lifschitz, Lehrbuch der theoretischen physik-Mechanik, Akademie-VerlagBerlin, 3rd edn., 1964, pp.101-104.

[11] J. G. Eisley, Nonlinear vibration of beams and rectangular plates, J. of Appl. Mathematics and Physics, 15 (1964), pp.9167-9175.

[12] S. Timoshenko, D. H. Young, W. Weaver, Jr., Vibration Problems in Engineering, John Wiley \& Sons, 4th edn., 1974, pp.177-186.

[13] J. P. Den Hartog, Mechanical Vibration, McGrawHill Book Company, Inc., 4th. edn., 1956, pp.370373

[14] C. Gui, R. Legtenberg, M. Elwenspoek and J. H. J. Fluitman, $Q$ factor dependence of one port encapsulated polysilicon resonator on reactive sealing pressure, Workshop Digest of MicroMechanics Europ(MME'94), Pisa, Italy, Scpt. $5-6$ 